\title{
Extracellular Matrix Controls Insulin Signaling in Mammary Epithelial Cells Through the RhoA/Rok Pathway
}

\author{
YI-JU LEE, ${ }^{*}$ TSAI-CHING HSU,' JYUN-YI DU,' ANTHONY J. VALENTIJN, ${ }^{2}$ TUNG-YI WU,' \\ CHENG-FU CHENG,' ZHIHONG YANG, ${ }^{3}$ AND CHARLES H. STREULI ${ }^{2 * *}$ \\ I'Institute of Immunology, Chung Shan Medical University, Taichung, Taiwan, Republic of China \\ ${ }^{2}$ Wellcome Trust Centre for Cell Matrix Research, Faculty of Life Sciences, University of Manchester, Manchester, United Kingdom \\ ${ }^{3}$ Vascular Biology, Institute of Physiology, University of Fribourg, Fribourg, Switzerland
}

\begin{abstract}
Cellular responses are determined by a number of signaling cues in the local microenvironment, such as growth factors and extracellular matrix (ECM). In cultures of mammary epithelial cells (MECs), functional differentiation requires at least two types of signal, lactogenic hormones (i.e., prolactin, insulin, and hydrocortisone) and the specialized ECM, basement membrane (BM). Our previous work has shown that ECM affects insulin signaling in mammary cells. Cell adhesion to BM promotes insulin-stimulated tyrosine phosphorylation of insulin receptor substrate-I (IRS-I) and association of PI3K with IRS-I, whereas cells cultured on stromal ECM are inefficient in transducing these post-receptor events. Here we examine the mechanisms underlying ECM control of IRS phosphorylation. Compared to cells cultured on BM, cells on plastic exhibit higher level of RhoA activity. The amount and the activity of Rho kinase (Rok) associated with IRS-I are greater in these cells, leading to serine phosphorylation of IRS-I. Expression of dominant negative RhoA and the application of Rok inhibitor Y27632 in cells cultured on plastic augment tyrosine phosphorylation of IRS-I. Conversely, expression of constitutively active RhoA in cells cultured on BM impedes insulin signaling. These data indicate that RhoA/Rok is involved in substratum-mediated regulation of insulin signaling in MECs, and under the conditions where proper adhesion to BM is missing, such as after wounding and during mammary gland involution, insulin-mediated cellular differentiation and survival would be defective.
\end{abstract}

J. Cell. Physiol. 220: 476-484, 2009. (c) 2009 Wiley-Liss, Inc.

Extracellular matrix (ECM) provides supportive, adhesive, and barrier functions for tissues, and also triggers specific signaling pathways and cytoskeleton reorganization. Various cellular responses, including proliferation, differentiation, migration, and survival are regulated by ECM. As these responses are also controlled by growth factors, cross-talk between ECM- and growth factor-activated signaling pathways has been proposed and documented (Giancotti and Tarone, 2003; Streuli and Akhtar, 2009). In this study, we explore possible mechanisms linking ECM to the insulin signaling pathway, using mammary epithelium as a model.

The breast is a glandular tissue composed of two types of epithelial unit: the collecting ducts, and the alveoli. The latter contain mammary epithelial cells (MECs) that adhere to a specialized ECM, the basement membrane (BM) in vivo. This interaction is necessary for the efficient activation of specific signaling pathways, for example those driven by prolactin and insulin, but not EGF (Edwards et al., I 998; Lee and Streuli, 1999; Wang et al., 2004). Recent findings have further revealed that $\beta I$ integrin is required for proper prolactin signaling, and Rac GTPase mediates this effect by decreasing the association of SHP-2 with Jak2 (Naylor et al., 2005; Akhtar and Streuli, 2006).

Culture studies have shown that insulin is critical for MEC differentiation and survival (Rosfjord and Dickson, 1999; Hadsell and Abdel-Fattah, 200I; Marshman and Streuli, 2002). Insulin initiates cascades of signaling via tyrosine phosphorylation of the insulin receptor (IR). The active receptor then recruits and phosphorylates Shc and the insulin receptor substrate (IRS), which contain multiple tyrosine residues and thus serve as docking proteins for downstream signaling molecules (Taniguchi et al., 2006). Several lines of evidence in a variety of cell systems have demonstrated that ECM regulates insulin/IGF-I signaling at different levels, thereby altering the intensity or duration of signaling.
First, ECM modulates the levels of insulin signaling components. In IR-overexpressing CHO-T cells, internalization of IR is decreased in cells adhered to galectin- 8 compared to those cultured on fibronectin, collagen, or laminin (Boura-Halfon et al., 2003). IRS-I expression is also modulated by adhesion; in DA2 fibroblasts this occurs at the transcriptional level, primarily through FAK (Lebrun et al., 2000). Second, integrins can regulate the phosphorylation of insulin/IGF-I signaling components by interacting with them. In $3 \mathrm{~T} 3$ and Rat I fibroblasts, adhesion promotes the association of $\alpha v \beta 3$ integrin with IR, while in $\mathrm{CHO}$ or $\mathrm{PC} 3$ cells overexpressing $\beta$ I integrin, $\beta$ I integrin interacts with IGF-I receptor (IGF-IR) and IRS-I in an IGF-I-dependent and independent manner, respectively (Schneller et al., 1997; Goel et al., 2004). In addition to $\alpha v \beta 3$ and $\beta$ I integrin, $\alpha 6$ integrin

Contract grant sponsor: National Health Research Institute; Contract grant numbers: NHRI-EX95-9304SC, NHRI-EX969304SC.

Contract grant sponsor: National Science Council; Contract grant number: NSC 92-2320-B-040-040.

*Correspondence to: Yi-Ju Lee, Institute of Immunology, Chung Shan Medical University, Taichung 402, Taiwan, Republic of China. E-mail: yijulee@csmu.edu.tw

**Correspondence to: Charles H. Streuli, Wellcome Trust Centre for Cell Matrix Research, Faculty of Life Sciences, University of Manchester, Manchester MI3 9PT, United Kingdom. E-mail: cstreuli@man.ac.uk

Received 4 September 2008; Accepted 13 March 2009

Published online in Wiley InterScience (www.interscience.wiley.com.), 23 April 2009. DOI: $10.1002 / j c p .21793$ 
associates with IGF-IR in embryonic lens cells in vivo (Walker et al., 2002). Interestingly, in T47D cells or MDA-MB-435 cells overexpressing $\beta 4$ integrin, engagement of $\alpha 6 \beta 4$ integrin itself induces tyrosine phosphorylation of IRS-I (Shaw, 200I). Third, tyrosine kinases downstream of integrin may be involved in insulin signaling, since FAK directly associates with IRS-I in 293 cells overexpressing FAK, and transient expression of FAK together with Src restores insulin-induced tyrosine phosphorylation of IR and IRS-I in suspended 3T3 cells that overexpress IR (Lebrun et al., I998; El Annabi et al., 200I). Finally, ECM can prevent the dephosphorylation of insulin/IGF-I signaling components. In smooth muscle cells, $\alpha v \beta 3$ integrin prolongs tyrosine phosphorylation of IGF-IR through a control on the rate of SHP-2 recruitment to the activated receptor (Maile and Clemmons, 2002). In pancreatic adenocarcinoma cell lines, MIA PaCa-2 and PANC-I, cell adhesion to fibronectin transactivates IGF-IR by preventing SHP-2 from dephosphorylating IGF-IR (Edderkaoui et al., 2007).

Many of these studies are restricted to overexpression analysis in a variety of cell and cancer lines, and no work has approached the mechanism of this cross-talk in normal epithelia. We have demonstrated that in primary cultures of normal MECs, insulin signaling propagates more effectively in cells plated on BM than in those on a stromal ECM represented by native collagen I or on tissue culture plastic (where cells adhere to fibronectin and vitronectin deposited from serum) (Farrelly et al., 1999; Lee and Streuli, 1999). Greater extents of IRS-I tyrosine phosphorylation and IRS-I/PI3K association take place in cells cultured on BM; however, levels of tyrosine phosphorylation of IR are comparable in cells on both substrata. We have therefore argued that ECM modulates insulin signaling downstream of the receptor at the level of IRS-I tyrosine phosphorylation.

In this study we have explored the underlying mechanisms of how ECM regulates insulin signaling, with an emphasis on the RhoA/Rho kinase (Rok) pathway and protein tyrosine phosphatases (PTPs), using primary cultures of MECs isolated directly from mammary gland.

\section{Materials and Methods Reagents}

Murine EGF, bovine insulin, and hydrocortisone were purchased from Sigma (St. Louis, MO). Antibody to Rok was obtained from Becton Dickinson (Bedford, MA). Antibody to phospho-IRS-I (Ser636/639) was from Cell Signaling (Beverly, MA). Antibodies to IR, RhoA, SHP-2, hemagglutinin (HA) and myosin phosphatase target subunit I (MYPTI) were from Santa Cruz Biotechnology (Santa Cruz, CA). Rho activation assay kit, recombinant MYPTI (654-880) and antibodies to phosphotyrosine (clone 4G I0), IRS- I and phospho-MYPTI were from Upstate Biotechnology (Lake Placid, NY). Y27632 was obtained from Calbiochem (San Diego, CA).

\section{Substrata and cell culture}

Reconstituted BM matrix, Matrigel, was purchased from Becton Dickinson and coated onto dishes at $7 \mathrm{mg} / \mathrm{ml}$. All experiments were performed with first or second passage MECs derived from mid-pregnant ICR mice. Primary epithelial cultures were prepared from isolated mammary alveoli and cultured in nutrient mixture F-I 2 (Sigma) containing I0\% fetal calf serum (Hyclone, Logan, UT), I mg/ml fetuin (Sigma), $5 \mathrm{ng} / \mathrm{ml}$ EGF and I $\mu \mathrm{g} / \mathrm{ml}$ hydrocortisone. The cells were plated onto Matrigel, serum-treated tissue culture plastic dishes (where the cells adhere to fibronectin and virtonectin), or $2 \mathrm{mg} / \mathrm{ml}$ collagen I renatured from HAc-dissolved rat tail collagen fibrils. After $72 \mathrm{~h}$, cells were serum-starved $6-8 \mathrm{~h}$ in Dulbecco's modified Eagle's medium/nutrient mixture F-I2
(Invitrogen, Grand Island, NY) containing hydrocortisone, and then subjected to various treatments.

\section{Adenovirus infection}

Recombinant adenovirus carrying HA-tagged dominant negative RhoA (NI9RhoA) and constitutively active RhoA (L63RhoA) were generated as previously described (Ming et al., 2002). For cells cultured on plastic, recombinant adenovirus was added to cells directly; whereas for those cultured on BM, cells were trypsinized, infected in suspension at $37^{\circ} \mathrm{C}$ for I h, and then plated onto Matrigel-coated dishes (Watkin and Streuli, 2002). After incubated with adenovirus for $18-24 \mathrm{~h}$, cells were serum-starved for $6-8 \mathrm{~h}$, and stimulated with insulin (100 nM) for $15 \mathrm{~min}$.

\section{Immunoprecipitation and Western blot analysis}

Untreated or growth factor-treated cells were lysed in lysis buffer containing $50 \mathrm{mM}$ Tris ( $\mathrm{pH} 7.4$ ), $150 \mathrm{mM} \mathrm{NaCl}, 2 \mathrm{mM}$ EDTA, I $\mathrm{mMNa}_{3} \mathrm{VO}_{4}, 10 \mathrm{mMNaF}, 10 \mu \mathrm{g} / \mathrm{ml}$ aprotinin, $10 \mu \mathrm{g} / \mathrm{ml}$ leupeptin, I mM phenylmethylsulfonyl fluoride, and I\% Triton X-I00. Normalization of protein was confirmed by SDS-PAGE, followed by Coomassie Blue staining. Cell lysates containing equal amounts of protein were incubated with I-2 $\mu \mathrm{g}$ of antibody and 20-50 $\mu$ l of protein A-Sepharose beads (Zymed Laboratories, Inc., South San Francisco, CA) overnight at $4^{\circ} \mathrm{C}$. Immunoprecipitates or whole cell lysates were subjected to SDS-PAGE, transferred to PVDF membrane (NEN), and probed with antibodies to anti-phosphotyrosine (4G I0; I $\mu \mathrm{g} / \mathrm{ml})$, IR ( $\mu \mathrm{g} / \mathrm{ml})$, IRS-I ( $\mu \mathrm{g} / \mathrm{ml})$, phospho-IRS- I (Ser636/639) (I:I,000), RhoA $(2 \mu \mathrm{g} / \mathrm{ml})$, HA (I $\mu \mathrm{g} / \mathrm{ml})$, Rok ( $\mathrm{\mu g} / \mathrm{ml})$, phospho-MYPTI $(2 \mu \mathrm{g} / \mathrm{ml})$, MYPTI (I $\mu \mathrm{g} / \mathrm{ml})$, and SHP-2 ( $\mathrm{\mu g} / \mathrm{ml}$ ). Proteins were visualized using an ECL kit (Cell Signaling). In each of the studies presented, the results shown are typical of three independent experiments.

\section{Rho activity assay}

Measurement of RhoA activation was performed according to manufacturer's instructions (Upstate Biotechnology). Briefly, cleared cell lysates were incubated for I h at $4{ }^{\circ} \mathrm{C}$ with GST-Rhotekin Rho-binding domain bound to glutathione-agarose beads to precipitate GTP-bound Rho. Total lysates and precipitates were analyzed by Western blotting using antibody to RhoA.

\section{Rok activity assay}

IRS-I associated Rok activity was measured according to manufacturer's instructions (Upstate Biotechnology) with minor modifications. Briefly, IRS-I immunoprecipitates were incubated with $500 \mathrm{ng}$ recombinant MYPTI (654-880) and $200 \mu$ M ATP (Cell Signaling) in kinase buffer (Cell Signaling) for $30 \mathrm{~min}$ at $30^{\circ} \mathrm{C}$. The reaction was stopped by adding Laemmli sample buffer, and then boiled for $5 \mathrm{~min}$. Phosphorylation of MYPTI was assessed by immunoblotting using antibody to phospho-MYPTI.

\section{In-gel PTP assay}

Experimental procedures followed the protocol reported by Burridge and Nelson (1995). Briefly, I mg of poly(glutamate/ tyrosine) (Sigma) was incubated with 500-I,000 $\cup$ of v-abl kinase (Calbiochem) and $500 \mu \mathrm{Ci}$ of $\left[\gamma-{ }^{32} \mathrm{P}\right]$ ATP in $0.5 \mathrm{ml}$ kinase buffer

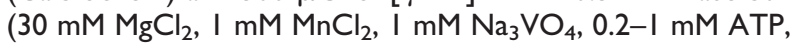
$10 \mathrm{mM}$ dithiothreitol, $0.05 \%$ Triton $X-100,50 \mathrm{mM}$ imidazole, $\mathrm{pH} 7.2$ ) at room temperature for $18 \mathrm{~h}$, and the reaction was terminated by addition of an equal volume of $20 \%$ trichloracetic acid. After $30 \mathrm{~min}$ on ice, the precipitated poly(glu/tyr) were sedimentated at $12,000 \mathrm{~g}$ for $10 \mathrm{~min}$ at $4^{\circ} \mathrm{C}$. The pellet was dissolved in $2 \mathrm{M}$ Tris-base, passed over a G50 Sephadex column equilibrated in $50 \mathrm{mM}$ imidazole, $\mathrm{pH} 7.2$, and collected in $0.5 \mathrm{ml}$ fractions. ${ }^{32} \mathrm{P}$-labeled substrate was added into the regular polyacrylamide gel mix prior to polymerization at $\sim 10^{5} \mathrm{cpm} / \mathrm{ml}$. Samples were separated by SDS-PAGE. Following electrophoresis, 
gels were first incubated in $50 \mathrm{mM}$ Tris- $\mathrm{HCl}, \mathrm{pH}$ 8.0, containing $20 \%$ isopropanol overnight to remove the SDS. Gels were then washed twice in $50 \mathrm{mM}$ Tris $-\mathrm{HCl}, \mathrm{pH}$ 8.0, containing $0.3 \% \beta$-mercaptoethanol, and denatured by incubating with the same buffer containing $6 \mathrm{M}$ guanidine hydrochloride and I mM EDTA for $90 \mathrm{~min}$. The gels were then incubated three times in renaturation buffer (I mM EDTA, 50 mM Tris- $\mathrm{HCl}$, $\mathrm{pH} 8.0$, $0.3 \% \beta$-mercaptoethanol, and $0.04 \%$ Tween- 40$)$. The gels were finally incubated overnight in renaturation buffer containing $4 \mathrm{mM}$ dithiothreitol. Following this incubation, the gels were stained with Coomassie brilliant blue, destained, and dried for autoradiography. The clear bands in a black background represent where PTPs localize.

\section{Results}

MECs cultured on plastic exhibit higher level of RhoA activity

Our previous work demonstrated that the nature of the substratum that MECs adhere to affects insulin signaling downstream of its receptor, at the level of IRS-I tyrosine phosphorylation (Farrelly et al., 1999; Lee and Streuli, 1999). The aim of this study was to further identify the mechanism for differential IRS activation in MECs grown on two substrata, tissue culture plastic where cells form monolayers and are poorly responsive to insulin, and BM where cells form three-dimensional acini and show robust insulin-activated signaling.

One approach to delineate the mechanism is to find out the differences in signaling pathways triggered by cell adhesion to plastic and BM, and then evaluates the involvement of these pathways in modulating insulin signaling. Among the pathways known to be activated by cell adhesion, we were particularly interested in Rho since its downstream effector Rok has been shown to regulate insulin signaling (Farah et al., 1998; Begum et al., 2002; Furukawa et al., 2005; Kanda et al., 2006; Lim et al., 2007). We found that MECs cultured on plastic displayed higher RhoA activity than those cultured on BM (Fig. I). In addition, when cells were cultured in monolayers on collagen I, there was a similar level of RhoA activity as in cells cultures on plastic (data not shown).

This result shows that activation of RhoA is differentially regulated by cell adhesion to different substrata.

\section{RhoA activity is detrimental for insulin signaling in MECs}

We then went on to examine whether RhoA accounted for the defectiveness of insulin signaling in MECs cultured on plastic. Lowering RhoA activity by expressing dominant negative RhoA (NI9RhoA) augmented insulin-induced IRS-I tyrosine

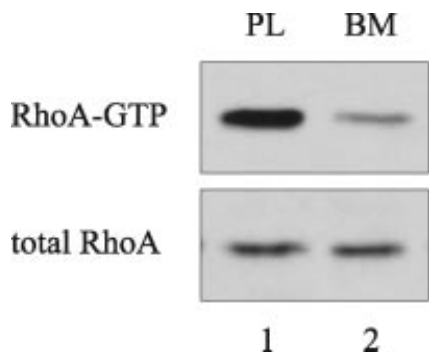

Fig. I. MECs cultured on plastic exhibit higher RhoA activity. MECs were cultured on plastic (PL) or BM. Cell lysates were incubated with GST-Rhotekin bound to glutathione-agarose beads to precipitate GTP-bound Rho. Total lysates and precipitates were then analyzed by immunoblotting using antibody to RhoA. phosphorylation by up to I.8-fold (Fig. 2B, lane 4 vs. lane 2, and Fig. 2C). In contrast, expression of constitutively active RhoA (L63RhoA) inhibited IRS-I tyrosine phosphorylation by $52 \%$ in MECs cultured on BM (Fig. 2E, lane 4 vs. lane 2, and Fig. 2F). Both treatments had marginal effects on IR tyrosine phosphorylation, but these were not statistically significant (Fig. 2A,C,D,F).

Collectively, these data suggest that culture on plastic, a substratum that is not suitable for optimal insulin signaling, activates RhoA, which in turn blocks insulin signaling in MECs.

\section{Rok binds and phosphorylates IRS-I in cells cultured on plastic}

A number of downstream effectors are activated by RhoA, including Rok. Rok is a serine/threonine kinase and is involved in several cellular responses, such as stress fiber formation, actinomyosin contraction, cell-substratum adhesion, cell-cell interaction, migration, cytokinesis, apoptosis, and gene expression (Riento and Ridley, 2003). One notable function of Rok is to regulate insulin/IGF-I signaling. It has been shown to bind and phosphorylate IRS-I, thereby suppressing tyrosine phosphorylation of IRS-I (Farah et al., 1998; Begum et al., 2002; Lim et al., 2007). Based on these findings, we examined the extent of association of Rok with IRS-I in MECs cultured on different substrata. A higher amount of Rok was co-immunoprecipitated with IRS-I in cells cultured on plastic (Fig. 3A), coinciding with greater Rok activity associated with IRS-I in these cells (Fig. 3B). The IRS-I -associated Rok activity in cells cultured on plastic was 3.6-fold more than that in cells cultured on BM (Fig. 3C).

Since Rok bound to IRS-I might directly phosphorylate IRS-I, we then measured IRS-I phosphorylation at serine residues 307,612 , and $636 / 639$ by immunoblotting. Serine phosphorylation of IRS-I at residues 307 and 612 was not detected in MECs regardless the substratum. However, the level of serine phosphorylation at residues 636/639 was higher in cells cultured on plastic (Fig. 4A), and this was diminished by the treatment of Y27632, a Rok inhibitor (Fig. 4B).

Our data thus suggest that higher RhoA activity in MECs cultured on plastic leads to greater association of Rok with IRS-I, resulting in phosphorylation at serine residues.

Inhibition of Rok activity by Y27632 increases tyrosine phosphorylation of IRS-I in MECs cultured on plastic

Serine phosphorylation of IRS-I exerts a negative impact on IRS-I tyrosine phosphorylation. Various serine/threonine kinases phosphorylate IRS-I, including PKC, Erk, JNK, IKK $\beta$, and Rok, as well as downstream effectors in the insulin-stimulated PI3K pathway, comprising GSK-3, mTOR, and p70S6K (reviewed by Zick, 200I; Johnston et al., 2003; Taniguchi et al., 2006). Given that greater extent of Rok binds and phosphorylates IRS-I in MECs cultured on plastic (Figs. 3 and 4), we examined whether inhibition of Rok activity by Y27632 could ameliorate insulin-induced tyrosine phosphorylation of IRS-I. Indeed, inclusion of Y27632 enhanced level of tyrosine phosphorylation of IRS-I in response to insulin by twofold in these cells (Fig. 5B, lane 3 vs. lane I), but did not affect tyrosine phosphorylation of IR (Fig. 5A). For cells cultured on BM, no significant effect of Y27632 on insulin signaling was observed (Fig. 5C,D), presumably because it was already maximal.

Taken together, our results suggest that the inefficiency of insulin signaling in MECs cultured on plastic is due to, at least in part, over-activation of RhoA/Rok pathway, leading to IRS-I serine phosphorylation and thus disruption of insulin signaling propagation downstream of IR. In contrast, MECs cultured on BM exhibit low level of RhoA, thereby acquiring full capacity of insulin signaling. 
A

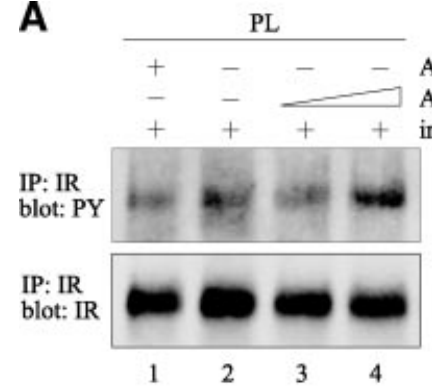

D

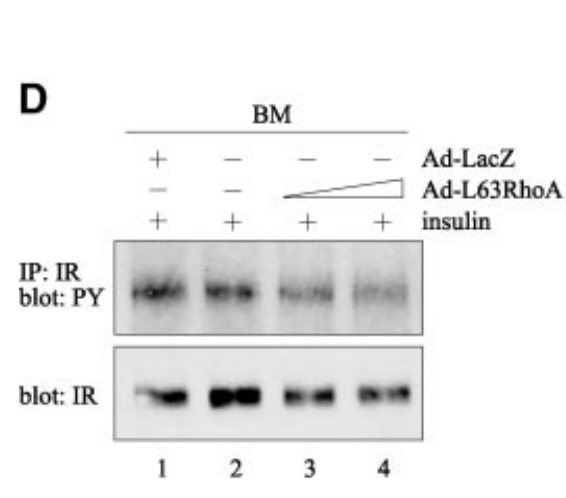

B

Ad-LacZ Ad-N19RhoA insulin

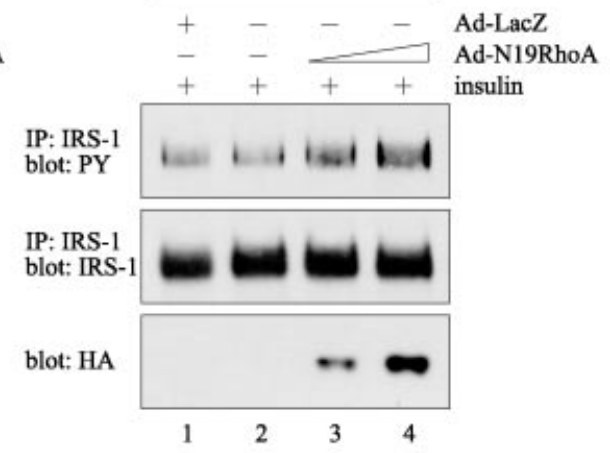

$\mathbf{E}$

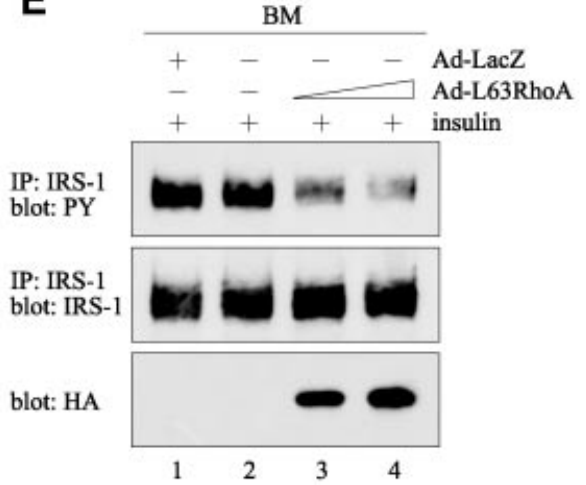

C

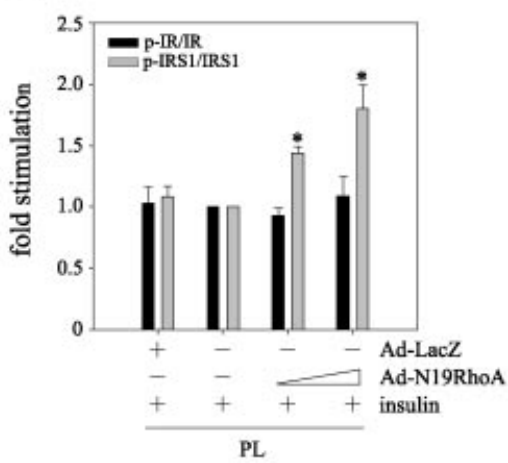

$\mathbf{F}$

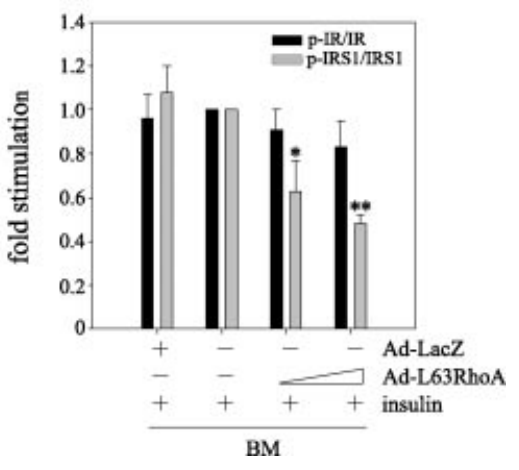

Fig. 2. Expression of dominant negative RhoA improves insulin signaling in MECs cultured on plastic, whereas expression of constitutively active RhoA inhibits insulin signaling in MECs cultured on BM. MECs cultured on plastic were infected with adenovirus carrying dominant negative RhoA (Ad-NI 9RhoA) or LacZ in situ (A,B). MECs cultured on BM were trypsinized, infected with adenovirus carrying constitutively active RhoA (Ad-L63RhoA) or LacZ, and replated on BM (D,E). After $24 \mathrm{~h}$, cells were serum-starved for $8 \mathrm{~h}$ and stimulated with I $00 \mathrm{nM}$ insulin for I 5 min. Cell lysates were immunoprecipitated (IP) with antibody to IR (A,D) or IRS-I (B,E), followed by immunoblotting with anti-phosphotyrosine antibody (PY). Blots were stripped and reprobed with the appropriate precipitating antibodies. Expression of HA was monitored by immunoblotting. C,F: Quantification of the effect of N I 9RhoA (C) and L63RhoA (F) on insulin signaling. Immunoblots from three independent experiments were analyzed by densitometry, and data are expressed as fold stimulation with respect to mock-infected cells. ${ }^{*} P<0.05$; ${ }^{* *} P<0.0$ I.

\section{PTPs association with IR and IRS-I}

Our previous work revealed that the PTP inhibitor, vanadate, elevates the tyrosine phosphorylation of IRS-I in MECs cultured on collagen I or plastic, although the mechanism was not determined (Lee and Streuli, 1999). Never-the-less, these data suggest that an additional mechanism for the ECM-mediated modulation of IRS-I tyrosine phosphorylation

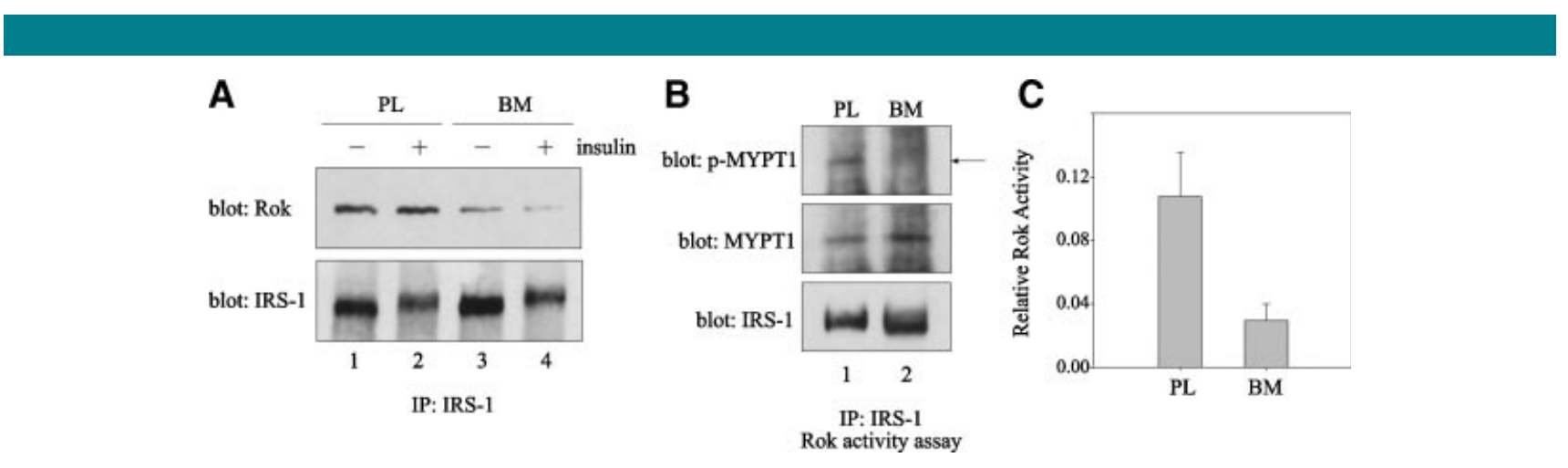

Fig. 3. The amount and activity of Rok associated with IRS-I are higher in MECs cultured on plastic. A: MECs cultured on plastic (PL) or BM were incubated in the absence or presence of $100 \mathrm{nM}$ insulin for $15 \mathrm{~min}$. Cell lysates were immunoprecipitated (IP) with antibody to IRS-I, followed by immunoblotting with anti-Rok antibody. The blot was then stripped and reprobed with antibody to IRS-I. B,C: Cell lysates were

immunoprecipitated with antibody to IRS-I followed by Rok activity assay using recombinant MYPTI as a substrate. The extent of MYPT I phosphorylation was monitored by immunoblotting with anti-phospho-MYPTI antibody. The blot was then stripped and repobed with antibody to MYPTI. IRS-I level in immunoprecipitates was also monitored by immunoblotting. C: Immunoblots of p-MYPTI and IRS-I were quantified by densitometric analysis. Relative Rok activity associated with IRS-I was calculated by dividing value of intensity of p-MYPT I by that of IRS-I. Data are expressed as mean $\pm \mathrm{SE}$ from three separate experiments. 
A

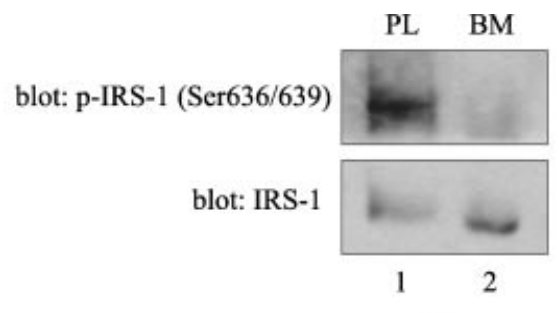

B

blot: p-IRS-1 (Ser636/639)

blot: IRS-1

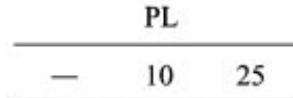

$\mathrm{Y} 27632(\mu \mathrm{M})$

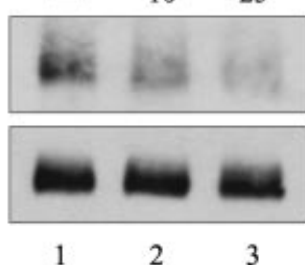

Fig. 4. MECs cultured on plastic exhibit higher extent of IRS-I serine phosphorylation, and this can be inhibited by Y27632. A: Total cell lysates obtained from MECs cultured on plastic (PL) and BM were analyzed by immunoblotting with antibodies to phospho-IRS-1

(Ser636/639) and IRS-I. B: MECs cultured on PL were pretreated without or with 10 or $25 \mu M$ Y27632 for I h, and cell lysates were analyzed by immunoblotting with antibodies to phospho-IRS-I (Ser636/639) and IRS-I. may involve a PTP. To search for possible PTPs associated with IRS-I or the receptor complex, an in-gel PTP assay was performed (Burridge and Nelson, 1995). Phosphorylated poly(glutamate-tyrosine) was incorporated into acrylamide gels, and samples were separated by SDS-PAGE. Following electrophoresis, gels were subjected to denaturation and then renaturation, and PTP activity was detected by autoradiography. The clear bands in a black background represent where PTPs localize. This method does not resolve membrane-associated PTPs, but is useful for possible identification of soluble PTPs.

The overall profile of PTPs from total cell lysates using the in-gel PTP assay was initially obtained. Similar patterns but different intensities of PTP were observed in MECs cultured on the two different substrata (Fig. 6). PTPs with a molecular weight of $\sim 130$ and $\sim 65 \mathrm{kDa}$ were predominant in cells cultured on plastic (asterisk), whereas those at the size of $\sim 35$ and $\sim 40 \mathrm{kDa}$ were detected mainly in cells cultured on BM (circle). Insulin stimulation did not alter the PTP profile. This result suggests that cell-ECM interaction may affect PTP expression or activity in MECs.

We then examined the possibility that adhesion to different substrata influences the association of PTPs with IRS-I or IR. Thus, IR and IRS-I immunoprecipitates were subjected to the in-gel PTP assay. Multiple PTPs coprecipitated with IR, but their levels did not change in response to insulin or the substratum (Fig. 7A). Notably, the migration profile of these PTPs on SDS gels is distinct to that of the overall PTP profile (compare Figs. 6 and 7), indicating that they represent a set of PTPs that associate specifically with IR. By contrast, insulin stimulated the association of a $\sim 70 \mathrm{kDa}$ PTP with IRS-I (Fig. 7B). This occurred

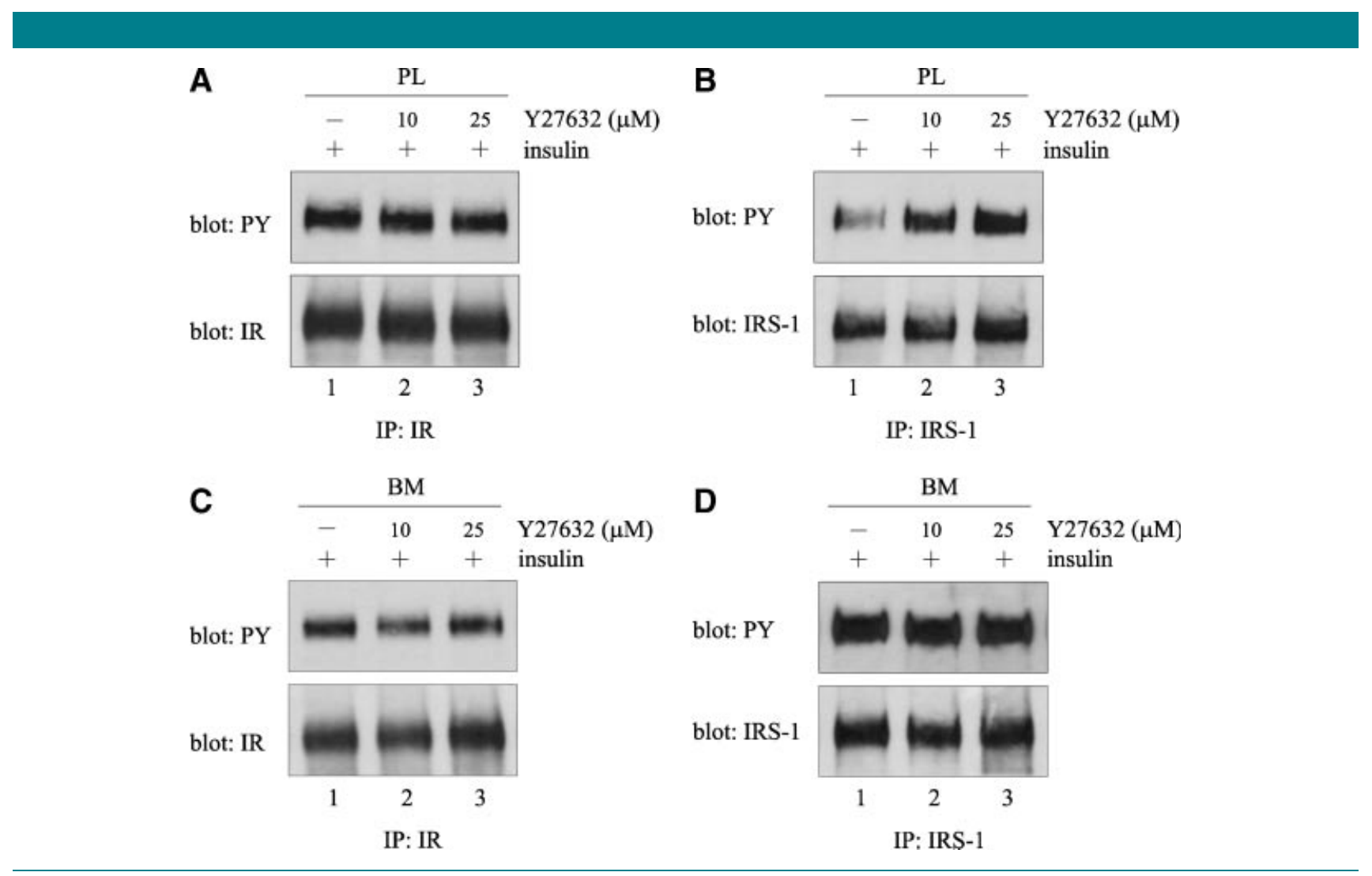

Fig. 5. Y27632 enhances insulin signaling in MECs cultured on plastic. MECs cultured on plastic(PL) (A,B) or BM(C,D) were pretreated without or with 10 or $25 \mu M$ Y27632 for I h, and then incubated with $100 \mathrm{nM}$ insulin for $15 \mathrm{~min}$. Cell lysates were immunoprecipitated (IP) with antibody to IR $(A, C)$ or IRS-I (B,D), followed by immunoblotting with anti-phosphotyrosine antibody (PY). Blots were stripped and reprobed with the appropriate precipitating antibodies. 


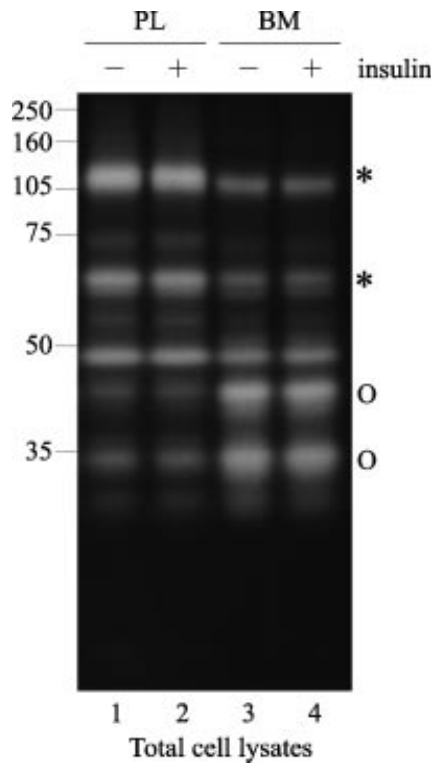

Fig. 6. The overall PTP expression profile is altered according to the substratum that MECs are cultured on. MECs cultured on plastic (PL) or BM were incubated in the absence and presence of $100 \mathrm{nM}$ insulin for 15 min. Total cell lysates were subjected to in-gel PTP assay. PTPs predominantly expressed in MECs cultured on plastic and $\mathrm{BM}$ are denoted by * and ${ }^{\circ}$, respectively.

in cells on both substrata, but the presence of this band was greater in cells cultured on BM (Fig. 7B, lane 2 vs. lane 4). This PTP may correspond to SHP-2 since it migrates at approximately the same size as the major band from SHP-2 immunoprecipitates (data not shown). To confirm this, immunoprecipitation of IRS-I followed by immunoblotting with SHP-2 antibody was carried out. In agreement with Figure 7B, a higher level of SHP-2 was associated with IRS-I upon insulin stimulation in cells cultured on BM (Fig. 7C).

The recruitment of SHP-2 to IRS-I requires IRS-I to be tyrosine phosphorylated first. We therefore hypothesized that more SHP-2 binds to IRS-I in MECs cultured on BM because of its higher level of tyrosine phosphorylation.

To test this possibility, we determined the effect of RhoA/Rok on insulin-induced recruitment of SHP-2 to IRS-I. Overexpression of constitutively active RhoA in MECs cultured on BM resulted in a decline in both IRS-I tyrosine phosphorylation and the extent of IRS-I/SHP-2 association (Fig. 8A). The converse experiment of including Y27632 in MECs cultured on plastic caused elevation of both tyrosine phosphorylation and SHP-2 binding (Fig. 8B). These results suggest that the recruitment of SHP-2 to IRS-I is not an upstream event controlling IRS-I tyrosine phosphorylation, but rather it is secondary to IRS-I tyrosine phosphorylation.

Thus, Rho/Rok exerts a negative effect on insulin signaling via a mechanism that does not involve SHP-2. Here we infer that SHP-2 might have a positive role in insulin signaling as reported by others (Maegawa et al., 1999).

\section{Discussion}

In MECs, insulin potentiates prolactin-induced milk protein gene expression and promotes cell survival (Farrelly et al., 1999; Lee and Streuli, 1999). These effects are apparent in cells cultured on BM, a substratum that is permissive for insulin signaling, but not in cells cultured on plastic dishes. We previously discovered that ECM regulates insulin signaling at the level of IRS-I tyrosine phosphorylation, and here we explore the mechanism of IRS regulation (Lee and Streuli, 1999). We demonstrate that cell adhesion to plastic activates RhoA and the downstream effector Rok, which then binds and phosphorylates IRS-I on serine residues, leading to inhibition of IRS-I tyrosine phosphorylation.

This differential activation of insulin signaling according to ECM contact is conceptually important because it explains in part why MECs survive and differentiate on BM, but are unable to do so when cultured on stromal ECM. Moreover, it has
A

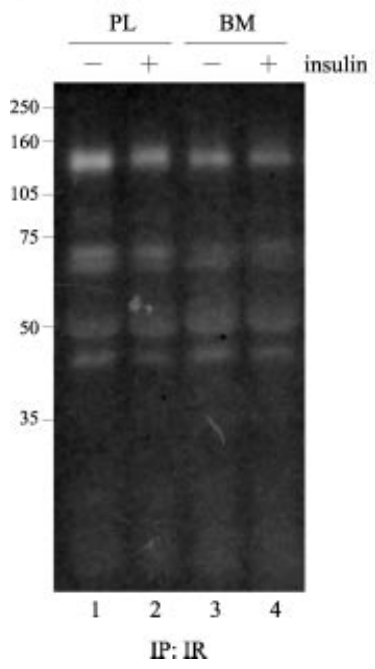

B

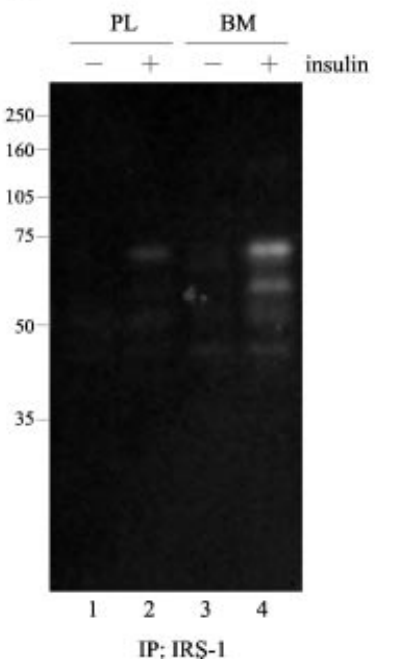

C

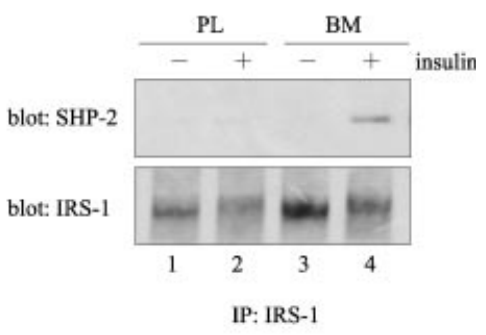

IP: IRS-1

Fig. 7. PTP association with IR and IRS-I. MECs cultured on plastic (PL) or BM were incubated in the absence or presence of $100 \mathrm{nM}$ insulin for 15 min. A,B: Total cell lysates were immunoprecipitated (IP) with antibody to IR (A) or IRS-I (B), followed by in-gel PTP assay. C: Total cell lysates were immunoprecipitated with anti-IRS-I antibody followed by immunoblotting with antibody to SHP-2. The blot was then stripped and reprobed with antibody to IRS-I. 
A

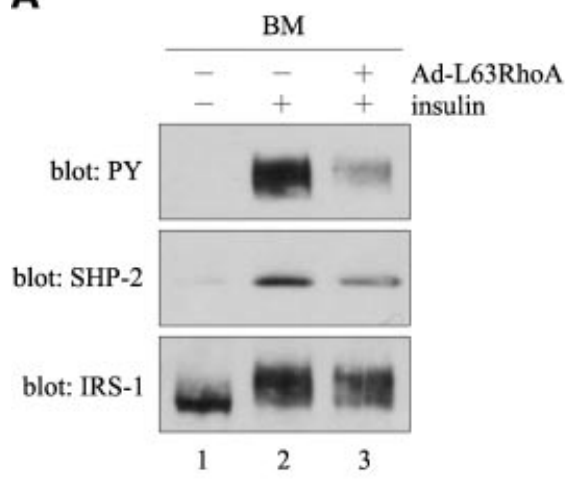

IP: IRS-1
B

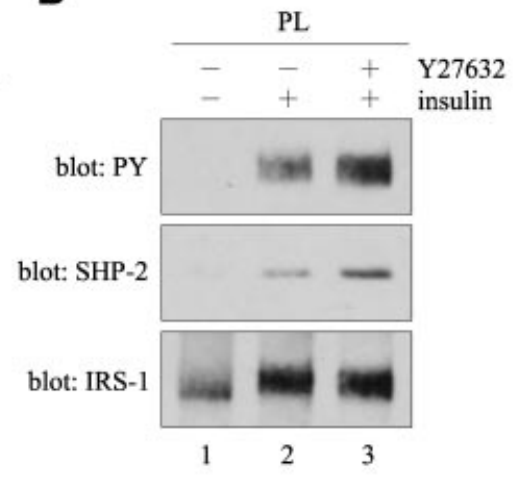

IP: IRS-1

Fig. 8. Insulin-induced recruitment of SHP-2 to IRS-I is repressed upon overexpressing constitutively active RhoA, but is augmented by Y27632 treatment. A: MECs cultured on BM were trypsinized, mock-infected or infected with adenovirus carrying constitutively active RhoA (Ad-L63RhoA), and replated on BM. After $24 \mathrm{~h}$, cells were serum-starved for $8 \mathrm{~h}$ and stimulated with I $00 \mathrm{nM}$ insulin for I 5 min. B: MECs cultured on plastic (PL) were serum-starved for $8 \mathrm{~h}$, pretreated without or with $15 \mu \mathrm{M}$ Y 27632 for I h, and then incubated with $100 \mathrm{nM}$ insulin for I 5 min. Total cell lysates were immunoprecipitated (IP) with antibody to IRS- I, followed by immunoblotting with anti-phosphotyrosine antibody (PY) and SHP-2. Blots were then stripped and reprobed with antibody to IRS-I.

developmental importance because if MECs, which normally contact BM in vivo, migrate into another ECM environment such as the subtending stroma, they undergo apoptosis. This is a key mechanism to maintain epithelial positioning within mature tissues and provides a strong selection to prevent inappropriate migration in malignancy (Streuli, 2006).

\section{RhoA is activated in culls cultured on plastic}

In our study, all experiments were performed in cells cultured on plastic or BM for at least 3 days. A striking difference between these cells is their morphology. MECs on plastic form a monolayer, whereas those on BM adopt an alveolar structure. Thus, it is conceivable that biochemical signals elicited by cell adhesion to these substrata are different. Indeed, our results indicate that RhoA is predominantly activated in cells cultured on plastic. Since MECs on BM still maintain the differentiated phenotype and survive but cells cultured on plastic do not, our result is consistent with previous observations that laminin-induced cell differentiation is accompanied by downregulation of RhoA activity (Gout et al., 200 I; Beqaj et al., 2002).

Another possibility for the differential activation of RhoA is the rigidity of the substratum. T47D breast epithelial cells cultured on plastic or 2D collagen exhibit higher level of RhoA than those on a 3D floating collagen gel (Wozniak et al., 2003). BM, like the 3D floating collagen gel, is pliable compared to the rigid surface of plastic dishes, and it has been proposed that cells exposed to malleable conditions do not need much RhoA activity to exert internal contractility because the opposing tension generated from cell adhesion is weaker (Olson, 2004). In addition to the composition and flexibility of the substratum, cell-cell interactions also influence RhoA activity, and vice versa. Cadherin engagement inhibits RhoA via tyrosine phosphorylation of pl90RhoGAP (Noren et al., 2003). Conversely, constitutive activation of RhoA disrupts tight junction and adherens junction in rat mammary epithelial tumor cells (Rubenstein et al., 2003). Therefore, cells cultured on BM have lower degree of RhoA activity since MECs require proper cell-cell junctions to achieve alveolar morphology and differentiation.

Thus a unifying explanation for the inefficient insulin signaling in MECs cultured in the conventional way (i.e., on tissue culture plastic) is that increased tension generated by adhesion to a rigid surface, leads to RhoA activation and the consequent Rok-induced inhibition of insulin signaling. Although such conditions do promote stress fiber formation and motility, which are important mediators of the wound-healing response, the same environment is not conducive for cellular differentiation and not ideal for epithelial cell survival.

Rok is activated in cells cultured on plastic and causes serine phosphorylation of IRS-I

Serine phosphorylation of IRS plays an important role in the control of insulin signaling. IRS phosphorylation mediated by PKB and $5^{\prime}$-AMP-activated protein kinase (AMPK) is beneficial to insulin signaling, whereas the effect of PKC, Erk, JNK, IKK $\beta$, GSK-3, mTOR, p70S6K, and Rok is detrimental (reviewed by Zick, 200I; Johnston et al., 2003; Taniguchi et al., 2006).

Regarding the latter, several mechanisms have been elucidated. Serine phosphorylation of IRS can cause dissociation of IRS from IR, suppression of IRS-I tyrosine phosphorylation, alteration of the subcellular location of IRS-I, augmentation of IRS degradation and inhibition of IR tyrosine kinase activity. All of these events lead to diminishment of insulin signaling. Here we found that MECs cultured on plastic exhibited higher extent of IRS-I serine phosphorylation (Fig. 4A). This might hinder the association of IRS-I with IR since we have observed that IR and IGF-IR form hybrid receptors in MECs, but the recruitment of IRS-I to the receptor complex in response to insulin is impaired in cells cultured on plastic (data not shown). Conceivably, downregulation of IRS-I tyrosine phosphorylation would thus take place (Lee and Streuli, 1999).

Among the serine/threonine kinases involved in IRS-I serine phosphorylation, Rok, a downstream effector of Rho (Riento and Ridley, 2003), is emerging as an important regulator of insulin/IGF-I signaling. A Xenopus homolog of Rok binds to the PTB domain of IRS-I, and microinjection of the mRNA corresponding to the C-terminus of Rok inhibits insulin-induced oocyte maturation (Farah et al., 1998). In vascular smooth muscle cells, Rok activation in response to thrombin results in an increase in its association with IRS-I, parallel with the enhancement of IRS-I serine phosphorylation and inhibition of insulin signaling (Begum et al., 2002). The role 
of Rok in insulin resistance has also been documented in several reports. Compared to the lean rats, RhoA/Rok activity and level of IRS-I seine phosphorylation are elevated in obese rats, coinciding with the reduction of insulin-induced signaling and responses (Kanda et al., 2006). Similar effects of Rok were observed in vascular endothelial cells because C-reactive protein, a molecule related to insulin resistance, activates RhoA/Rok/JNK via spleen tyrosine kinase (Syk), rendering serine phosphorylation of IRS-I and suppression of insulin responses (Xu et al., 2007). During muscle differentiation and adipogenesis mediated by insulin and IGF-I, a decline of RhoA/Rok activity occurs and inhibition of Rok facilitates differentiation. Likewise, upregulation of IRS-I serine phosphorylation and downregulation of insulin/IGF-I signaling are associated with higher Rok activity (Lim et al., 2007; Noguchi et al., 2007).

In concordance with these studies, we demonstrate here that RhoA/Rok is involved in substratum-mediated regulation of insulin signaling in MECs, and it exerts an adverse effect in cells that lack adherence to BM.

\section{The Rho pathway and mammary differentiation}

RhoGAP negatively regulates Rho activity. In p 190-B RhoGAP-deficient mice, elevated RhoA activity results in Rok-mediated IRS-I serine phosphorylation and the interruption of IGF-I signaling, thereby reducing cell and organ size as well as promoting adipogenesis rather than myogenesis from mesenchymal stem cells (Sordella et al., 2002, 2003). Defective embryonic mammary bud development and adult ductal morphogenesis, caused by decreased cell proliferation, have been observed in these mice, and these features are phenocopied in IGF-IR and IRS-I/2 knockout mice (Chakravarty et al., 2003; Heckman et al., 2007). However, in the mammary glands of p $190-\mathrm{B}$ overexpressing mice, ductal morphogenesis is impaired and hyperplastic lesions is induced, suggesting that a fine tuning of Rho activity is required for proper mammary gland development (Vargo-Gogola et al., 2006).

Collectively, these studies demonstrate the role of RhoGAP in development and differentiation through a control on IGF-I/insulin signaling. This is consistent with our and other observations that RhoA/Rok inhibits IGF-l/insulin signaling (Farah et al., 1998; Begum et al., 2002; Kanda et al., 2006; Lim et al., 2007; Noguchi et al., 2007; Xu et al., 2007).

One function of insulin is to cooperate with other lactogenic hormones, prolactin and hydrocortisone, to promote milk synthesis. In further studies, we have examined the effect of RhoA/Rok on prolactin signaling and the expression a milk protein gene, $\beta$-casein. As is the case with insulin signaling, we found that RhoA/Rok had an inhibitory effect on prolactin signaling (manuscript in preparation). We therefore suggest that, in the absence of proper cell-BM interactions, an over-activated RhoA/Rok pathway suppresses both insulin and prolactin signaling, culminating in a milk deficiency and reduced differentiation.

\section{IRS-I associates with SHP-2}

A further mechanism for regulating insulin signaling is through PTPs (Lee and Streuli, 1999). Here we examined PTPs targeting to IR and IRS-I, particularly those whose effect was regulated by ECM, initially with the aim of identifying PTPs that might suppress insulin signaling in cells cultured on plastic. However, we were unable to identify the association of specific PTPs with IRS-I under these conditions. This suggests that the low levels of tyrosine phosphorylation on IRS-I are caused via a different mechanism, which is likely to be the elevated serine phosphorylation caused by Rok.
Using an in-gel assay, we found that several PTPs were associated with IR but the extent of association was comparable in cells cultured on plastic and BM (Fig. 7A). This is in agreement with our previous result that insulin stimulates tyrosine phosphorylation of IR irrespective of substratum (Lee and Streuli, 1999). On the other hand, coprecipitation of SHP-2 and IRS-I in response to insulin was more prominent in cells cultured on BM (Fig. 7B,C). This might be as a result of elevated IRS-I tyrosine phosphorylation (Lee and Streuli, 1999). Furthermore, we found that the effect of RhoA/Rok on IRS-I/SHP-2 association was similar to that on IRS-I tyrosine phosphorylation (Fig. 8), indicating that SHP-2 is not involved in RhoA/Rok-mediated inhibition of insulin signaling. Here we suggest that SHP-2 might have a positive role in insulin signaling. This agrees with previous studies where IRS-I tyrosine phosphorylation and downstream signaling are attenuated in transgenic mice expressing dominant negative SHP-2 (Maegawa et al., 1999). The underlying mechanism for the positive effect of SHP-2 on signaling likely occurs through dephosphorylating docking sites for negative regulators (Ali et al., 2003; Neel et al., 2003). Although our data suggest that SHP-2 does not play a role in matrix-mediated control of insulin signaling, the possibility that other PTPs are involved cannot be excluded.

\section{Summary}

We have dissected some aspects of the mechanism by which cell-ECM interactions control insulin signaling in mammary gland. We find that cell adhesion to different substrata differentially activates the RhoA/Rok pathway, which then controls signal propagation downstream of IR by a mechanism involving serine phosphorylation of IRS-I. Thus, the ECM exerts an intricate control on the ability of MECs to receive and respond to insulin signals, so that adhesion to a permissive BM promotes signaling while it is prevented on stromal matrix. It is likely that the tension of cytoskeleton, organization of cell interior, compartmentalization of signaling molecules, and even programming of gene expression are altered by different microenvironmental constraints on cells. Perhaps the integration of all these effects confers the ultimate selectivity in insulin signaling.

\section{Acknowledgments}

This work was funded by the National Health Research Institute (NHRI-EX95-9304SC, NHRI-EX96-9304SC) and National Science Council (NSC 92-2320-B-040-040). CHS is funded by the Wellcome Trust.

\section{Literature Cited}

Akhtar N, Streuli CH. 2006. Racl links integrin-mediated adhesion to the control of lactational differentiation in mammary epithelia. J Cell Biol 173:78I-793.

Ali S, Nouhi Z, Chughtai N. 2003. SHP-2 regulates SOCS-I-mediated Janus kinase-2 ubiquitination/degradation downstream of the prolactin receptor. J Biol Chem 278:5202I-52031.

Begum N, Sandu OA, Ito M, Lohmann SM, Smolenski A. 2002. Active Rho kinase (ROK-alpha) associates with insulin receptor substrate-I and inhibits insulin signaling in vascular smooth muscle cells. Jiol Chem 277:6214-6222.

Beqaj S, Jakkaraju S, Mattingly RR, Pan D, Schuger L. 2002. High RhoA activity maintains the undifferentiated mesenchymal cell phenotype, whereas RhoA down-regulation by laminin-2 induces smooth muscle myogenesis. J Cell Biol I56:893-903.

Boura-Halfon S, Voliovitch H, Feinstein R, Paz K, Zick Y. 2003. Extracellular matrix proteins modulate endocytosis of the insulin receptor. J Biol Chem 278:16397-16404.

Burridge K, Nelson A. 1995. An in-gel assay for protein tyrosine phosphatase activity: Detection of widespread distribution in cells and tissues. Anal Biochem 232:56-64.

Chakravarty G, Hadsell D, Buitrago W, Settleman J, Rosen JM. 2003. p I90-B RhoGAP Chakravarty G, Hadsell D, Buitrago W, Settleman J, Rosen JM. 2003. pl90-B
regulates mammary ductal morphogenesis. Mol Endocrinol 17:1054-1065.

Edderkaoui M, Hong P, Lee JK, Pandol SJ, Gukovskaya AS. 2007. Insulin-like growth factorreceptor mediates the prosurvival effect of fibronectin. J Biol Chem 282:26646-26655.

Edwards GM, Wilford FH, Liu X, Hennighausen L, Djiane J, Streuli CH. 1998. Regulation of mammary differentiation by extracellular matrix involves protein-tyrosine phosphatases. J Biol Chem 273:9495-9500.

El Annabi S, Gautier N, Baron V. 200I. Focal adhesion kinase and Src mediate integrin regulation of insulin receptor phosphorylation. FEBS Lett 507:247-252.

Farah S, Agazie Y, Ohan N, Ngsee JK, Liu XJ. 1998. A rho-associated protein kinase, ROKalpha, binds insulin receptor substrate-I and modulates insulin signaling. J Biol Chem 273:4740-4746. 
Farrelly N, Lee YJ, Oliver J, Dive C, Streuli CH. 1999. Extracellular matrix regulates apoptosis in mammary epithelium through a control on insulin signaling. J Cell Biol I44:1337-1348.

Furukawa N, Ongusaha P, Jahng W], Araki K, Choi CS, Kim HJ, Lee YH, Kaibuchi K, Kahn BB, Masuzaki H, Kim JK, Lee SW, Kim YB. 2005. Role of Rho-kinase in regulation of insulin action and glucose homeostasis. Cell Metab 2:119-129.

Giancotti FG, Tarone G. 2003. Positional control of cell fate through joint integrin/receptor protein kinase signaling. Annu Rev Cell Dev Biol 19:173-206.

Goel HL, Fornaro M, Moro L, Teider N, Rhim JS, King M, Languino LR. 2004. Selective modulation of type I insulin-like growth factor receptor signaling and functions by betal integrins. J Cell Biol 166:407-4I8.

Gout SP, Jacquier-Sarlin MR, Rouard-Talbot L, Rousselle P, Block MR. 200I. RhoAdependent switch between alpha2betal and alpha3betal integrins is induced by laminin-5 during early stage of HT-29 cell differentiation. Mol Biol Cell I2:3268-328I.

Hadsell DL, Abdel-Fattah G. 200I. Regulation of cell apoptosis by insulin-like growth factor I. Adv Exp Med Biol 501:79-85.

Heckman BM, Chakravarty G, Vargo-Gogola T, Gonzales-Rimbau M, Hadsell DL, Lee AV, Settleman J, Rosen JM. 2007. Crosstalk between the p190-B RhoGAP and IGF signaling pathways is required for embryonic mammary bud development. Dev Biol 309:137-|49.

Johnston AM, Pirola L, Van Obberghen E. 2003. Molecular mechanisms of insulin receptor substrate protein-mediated modulation of insulin signalling. FEBS Lett 546:32-36.

Kanda T, Wakino S, Homma K, Yoshioka K, Tatematsu S, Hasegawa K, Takamatsu I, Sugano N, Hayashi K, Saruta T. 2006. Rho-kinase as a molecular target for insulin resistance and hypertension. FASEB J 20:169-171.

Lebrun P, Mothe-Satney I, Delahaye L, Van Obberghen E, Baron V. 1998. Insulin receptor substrate-I as a signaling molecule for focal adhesion kinase pp 2 (FAK) and pp60(src). Biol Chem 273:32244-32253.

Lebrun P, Baron V, Hauck CR, Schlaepfer DD, Van Obberghen E. 2000. Cell adhesion and focal adhesion kinase regulate insulin receptor substrate-I expression. J Biol Chem 275:3837I-38377.

Lee YJ, Streuli CH. 1999. Extracellular matrix selectively modulates the response of mammary epithelial cells to different soluble signaling ligands. J Biol Chem 274:2240I-22408.

Lim MJ, Choi KJ, Ding Y, Kim JH, Kim BS, Kim YH, Lee J, Choe W, Kang I, Ha J, Yoon KS, Kim SS. 2007. RhoA/Rho kinase blocks muscle differentiation via serine phosphorylation of insulin receptor substrate-I and -2. Mol Endocrinol 21:2282-2293.

Maegawa H, Hasegawa M, Sugai S, Obata T, Ugi S, Morino K, Egawa K, Fujita T, Sakamoto T, Nishio Y, Kojima H, Haneda M, Yasuda H, Kikkawa R, Kashiwagi A. 1999. Expression of a dominant negative SHP-2 in transgenic mice induces insulin resistance. J Biol Chem 274:30236-30243.

Maile LA, Clemmons DR. 2002. The alphaVbeta3 integrin regulates insulin-like growth factor (IGF-I) receptor phosphorylation by altering the rate of recruitment of the Src-homology 2-containing phosphotyrosine phosphatase-2 to the activated IGF-I receptor. Endocrinology 143:4259-4264.

Marshman E, Streuli CH. 2002. Insulin-like growth factors and insulin-like growth factor binding proteins in mammary gland function. Breast Cancer Res 4:23I-239.

Ming XF, Viswambharan H, Barandier C, Ruffieux J, Kaibuchi K, Rusconi S, Yang Z. 2002. Rho GTPase/Rho kinase negatively regulates endothelial nitric oxide synthase phosphorylation through the inhibition of protein kinase B/Akt in human endothelial cells. Mol Cell Biol 22:8467-8477.

Naylor MJ, Li N, Cheung J, Lowe ET, Lambert E, Marlow R, Wang P, Schatzmann F, Wintermantel T, Schuetz G, Clarke AR, Mueller U, Hynes NE, Streuli CH. 2005. Ablation of betal integrin in mammary epithelium reveals a key role for integrin in glandula morphogenesis and differentiation. J Cell Biol 171:717-728.
Neel BG, Gu H, Pao L. 2003. The 'Shp'ing news: SH2 domain-containing tyrosine phosphatases in cell signaling. Trends Biochem Sci 28:284-293.

Noguchi M, Hosoda K, Fujikura J, Fujimoto M, Iwakura H, Tomita T, Ishii T, Arai N, Hirata M, Ebihara K, Masuzaki H, Itoh H, Narumiya S, Nakao K. 2007. Genetic and pharmacological inhibition of Rho-associated kinase II enhances adipogenesis. J Biol Chem 282:29574-29583

Noren NK, Arthur WT, Burridge K. 2003. Cadherin engagement inhibits RhoA via pl90RhoGAP. J Biol Chem 278:13615-13618.

Olson MF. 2004. Contraction reaction: Mechanical regulation of Rho GTPase. Trends Cell Biol |4:III-I|4.

Riento K, Ridley AJ. 2003. Rocks: Multifunctional kinases in cell behaviour. Nat Rev Mol Cell Biol 4:446-456.

Rosfjord EC, Dickson RB. 1999. Growth factors, apoptosis, and survival of mammary epithelial cells. J Mammary Gland Biol Neoplasia 4:229-237.

Rubenstein NM, Guan Y, Woo PL, Firestone GL. 2003. Glucocorticoid down-regulation of RhoA is required for the steroid-induced organization of the junctional complex and tight junction formation in rat mammary epithelial tumor cells. J Biol Chem 278:10353-10360.

Schneller M, Vuori K, Ruoslahti E. 1997. Alphavbeta3 integrin associates with activated insulin and PDGFbeta receptors and potentiates the biological activity of PDGF. EMBO 16:5600-5607.

Shaw LM. 200I. Identification of insulin receptor substrate I (IRS-I) and IRS-2 as signaling intermediates in the alpha6beta4 integrin-dependent activation of phosphoinositide 3-OH kinase and promotion of invasion. Mol Cell Biol 21:5082-5093.

Sordella R, Classon M, Hu KQ, Matheson SF, Brouns MR, Fine B, Zhang L, Takami H, Yamada Y, Settleman J. 2002. Modulation of CREB activity by the Rho GTPase regulates cell and organism size during mouse embryonic development. Dev Cell 2:553-565.

Sordella R, Jiang W, Chen GC, Curto M, Settleman J. 2003. Modulation of Rho GTPase signaling regulates a switch between adipogenesis and myogenesis. Cell II3:147-I58.

Streuli CH. 2006. Cell adhesion and cancer. In: Pelengaris S, Kahn M, editors. The molecular biology of cancer. Malden, USA: Blackwell Publishing Inc. p. 356-387.

Streuli CH, Akhtar N. 2009. Signal co-operation between integrins and other receptor systems. Biochem J 4I8:49|-506.

Taniguchi CM, Emanuelli B, Kahn CR. 2006. Critical nodes in signalling pathways: Insights into insulin action. Nat Rev Mol Cell Biol 7:85-96.

Vargo-Gogola T, Heckman BM, Gunther EJ, Chodosh LA, Rosen JM. 2006. PI90-B Rho GTPase-activating protein overexpression disrupts ductal morphogenesis and induces hyperplastic lesions in the developing mammary gland. Mol Endocrinol 20:139|-|405.

Walker JL, Zhang L, Zhou J, Woolkalis MJ, Menko AS. 2002. Role for alpha 6 integrin during lens development: Evidence for signaling through IGF-IR and ERK. Dev Dyn 223:273-284.

Wang P, Gilmore AP, Streuli CH. 2004. Bim is an apoptosis sensor that responds to loss of survival signals delivered by epidermal growth factor but not those provided by integrins. Biol Chem 279:41280-4I285.

Watkin H, Streuli CH. 2002. Adenoviral-mediated gene transfer in two-dimensional and three-dimensional cultures of mammary epithelial cells. Methods Cell Biol 69:403-423.

Wozniak MA, Desai R, Solski PA, Der CJ, Keely PJ. 2003. ROCK-generated contractility regulates breast epithelial cell differentiation in response to the physical properties of a three-dimensional collagen matrix. J Cell Biol 163:583-595.

Xu JW, Morita I, Ikeda K, Miki T, Yamori Y. 2007. C-reactive protein suppresses insulin signaling in endothelial cells: Role of spleen tyrosine kinase. Mol Endocrinol 21:564-573.

Zick Y. 200I. Insulin resistance: A phosphorylation-based uncoupling of insulin signaling. Trends Cell Biol II:437-44I. 